\title{
Distúrbios Urinários no Climatério: Avaliação Clínica e Urodinâmica
}

\author{
Postmenopausal Urinary Disorders: Clinical And Urodynamic Evaluation
}

\author{
João Paulo Sartori, Fernando T. Kawakami, Marair G.F. Sartori, \\ Manoel J.B.C. Girão, Edmund C. Baracat, Geraldo R. de Lima
}

\begin{abstract}
RESUMO
Objetivos: avaliar a freqüência dos distúrbios urinários e a variação dos parâmetros urodinâmicos segundo o tempo de pós-menopausa.

Métodos: foram estudadas 242 mulheres menopausadas atendidas nos Setores de Climatério e de Uroginecologia e Cirurgia Vaginal da Disciplina de Ginecologia da Escola Paulista de Medicina, UNIFESP, que apresentavam queixas urinárias. As pacientes foram agrupadas segundo o tempo de pós-menopausa em: grupo $A$ - até 4 anos; grupo $B$ - de 5 a 9 anos $e$ grupo $C$ - mais de 10 anos. Todas foram submetidas a anamnese, exame ginecológico $e$ estudo urodinâmico. Analisamos a freqüência de alterações urinárias e a variação dos parâmetros urodinâmicos, como volume urinário (VOL); tempo total de micção (TTM); fluxos urinário máximo (FMAX) e médio (FM); resíduo pós-miccional (RES); capacidade vesical no primeiro desejo miccional (CV1D); capacidade vesical máxima (CVM); pressão máxima de fechamento uretral e comprimento funcional da uretra, com bexiga cheia e vazia (PMCH, $P M V, C F U C H, C F V)$. Os dados foram analisados estatisticamente.

Resultados: o diagnóstico clínico mais comum foi de incontinência urinária de esforço (IUE) nos três grupos, porém observou-se maior incidência de urgência miccional com o evoluir do tempo de pós-menopausa. Em relação ao diagnóstico urodinâmico, 93,6\%, 84,6\% e 90,7\%, respectivamente, das pacientes dos grupos $A, B$ e $C$ apresentaram IUE, ao passo que 4,8\%, $13,5 \%$ e 6,2\% revelaram instabilidade vesical. Houve diminuição dos seguintes parâmetros urodinâmicos, segundo o tempo de pós-menopausa: TTM, FMAX e CV1D, além de aumento do residuo pós-miccional.

Conclusões: apesar da elevada incidência de sintomas urinários irritativos, como urgência incontinência, a IUE foi a principal afecção urinária nesta faixa etária.
\end{abstract}

PALAVRAS-CHAVE: Climatério. Distúrbios urinários. Urodinâmica. Incontinência urinária.

\section{Introdução}

A incontinência urinária é um dos principais problemas que acomete as mulheres na pósmenopausa ${ }^{15,19,25}$. Trata-se de afecção de grande impacto social, econômico e psicológico, sendo

Setor de Uroginecologia e Cirurgia Vaginal da Disciplina de Ginecologia da Escola Paulista de Medicina, Universidade Federal de São Paulo, São Paulo, SP, Brasil.

Correspondência:

João Paulo Sartori

Av. Onze de Junho, 1006 ap. 51 - Vila Mariana

04041-003 - São Paulo - SP - Brasil muitas vezes incapacitante. Nas últimas décadas, com o aumento da expectativa de vida da população, a mulher passa cerca de um terço da sua vida em situação de hipoestrogenismo, sofrendo as suas eventuais conseqüências ${ }^{1,2,3,22}$.

No trato urogenital, o estrogênio desempenha importante papel no mecanismo de continência urinária. Atua, por exemplo, aumentando o trofismo da mucosa da uretra, favorecendo a coaptação das paredes uretrais através das dobras de mucosa ${ }^{11,12,14}$. Tem ação, ainda, no tônus da musculatura pélvica, pelo aumento do número e da sensibilidade dos receptores alfa-adrenérgicos ${ }^{9}$, 
da vascularização uretral ${ }^{21,23}$ e do tecido conjuntivo periuretral, estimulando os fibroblastos a produzirem o colágeno ${ }^{8}$. É evidente, portanto, que a diminuição estrínica na pós-menopausa favorece a incontinência urinária de esforço.

Antes da utilização do exame urodinâmico, diversos autores já observavam melhora da perda de urina após a administração de estrogênios ${ }^{5,19,20,24,25,26,}$. Ressalta-se, porém, que a falta de padronização do diagnóstico da perda de urina, das doses, tipos e vias de administração de estrogênios dificultam sobremaneira a comparação dos resultados ${ }^{10}$.

Com a realização do exame urodinâmico passou-se a obter dados mais fiéis e de melhor reprodução ${ }^{6,13}$. Em 1979, Henriksson et al. ${ }^{17}$ estudando diversas faixas etárias, observaram queda significante da pressão de fechamento uretral e do comprimento funcional da uretra com o avançar da idade.

$\mathrm{Rud}^{23}$ relatou aumento progressivo da pressão uretral desde a infância até a adolescência, com diminuição após os 25 anos. Referiu, ainda, ser o comprimento funcional da uretra menor no climatério. A diminuição da pressão de fechamento uretral e do comprimento funcional da uretra na pós-menopausa foi referida também por outros autores ${ }^{7,13,27}$.

Segundo Diokno et al. ${ }^{13}$ e Gillon et al. ${ }^{16}$, há diminuição da capacidade vesical e aumento do resíduo urinário a medida que a idade aumenta, sendo que $37 \%$ da pacientes idosas não suportam mais que $300 \mathrm{ml}$ de volume. Em nosso meio, Wakavaiachi ${ }^{28}$ observou diminuição da capacidade vesical no primeiro desejo miccional e da pressão máxima de fechamento uretral somente em mulheres incontinentes com menos de 5 anos de pósmenopausa, quando comparadas às continentes.

Assim, procurou-se, no presente estudo, avaliar pacientes na pós-menopausa com perda de urina, identificando quais os tipos de incontinência urinária mais freqüentes, bem como a eventual variação dos parâmetros urodinâmicos.

\section{Pacientes e Métodos}

Foram incluídas no estudo 242 pacientes na menopausa com queixas urinárias, atendidas no Setores de Uroginecologia e Cirurgia Vaginal e de Climatério da Disciplina de Ginecologia da Escola Paulista de Medicina - Universidade Federal de São Paulo, no período de 1993 a 1996. Incluíram-se aquelas em amenorréia superior a um ano ou com niveis séricos de gonadotrofinas compativeis com o estado menopausal. A seguir, foram divididas em três grupos, segundo o tempo de pós-menopausa, isto é, grupo A: até 5 anos; grupo B: 5 a 10 anos e grupo C: mais do que 10 anos.

Todas as pacientes submeteram-se a anamnese e exame ginecológico, para caracterizar o padrão da perda de urina, utilizando-se a posição ortostática, quando necessário.

Estabeleceu-se o diagnóstico clínico de incontinência urinária de esforço quando havia queixa de perda de urina sincrônica ao esforço, na ausênca de outros sintomas irritativos, como disúria, polaciúria ou urgência miccional. O exame ginecológico complementou o diagnóstico, com a comprovação objetiva da perda de urina no momento do esforço solicitado e a avaliação do grau de distopia urogenital.

Nos casos em que a queixa foi de perda de urina associada a urgência miccional ou incontinência não-relacionada aos esforços, com ou sem lesão anatômica ou perda de urina evidentes, fez-se o diagnóstico clínico de incontinência de urgência

Finalmente, quando os sintomas estavam mesclados, considerou-se como incontinência urinária mista.

Todas foram submetidas a exame urodinâmico, constituído por fluxometria, cistometria, com água destilada a temperatura ambiente, e perfil pressórico uretral com cateter de fluxo. As definições, métodos e unidades adotadas são as padronizadas pela Sociedade Internacional de Continência ${ }^{4,18}$.

O diagnóstico urodinâmico foi de incontinência urinária de esforço quando se notou perda de urina simultânea ao esforço solicitado, durante a fase de enchimento vesical, sem atividade do detrusor. O diagnóstico foi de instabilidade do detrusor quando se registraram contrações do músculo com mais de $15 \mathrm{cmH}_{2} \mathrm{O}$ ou de menor amplitude, porém acompanhada de perda de urina e de incontinência urinária mista, quando houve associação de ambas as situações.

Foram avaliados o volume urinário $(\mathrm{V})$, o fluxo urinário médio (FM) e o máximo (FMAX), o resíduo pós-miccional (RES), a capacidade vesical no primeiro desejo miccional (CV1D), a capacidade vesical máxima (CVM), a pressão de fechamento uretral (PFU) e o comprimento funcional da uretra ( $\mathrm{CFU}$ ).

\section{Resultados}

No grupo A foram incluídas $62(25,6 \%)$ pacientes, $52(21,5 \%)$ no grupo B e $128(52,9 \%)$ no grupo C. Os dados referentes à idade, ao número de gestações, de partos normais ou de cesárias encontram-se na Tabela 1.

Apresentamos na Tabela 2, o diagnóstico clínico e, na Tabela 3, o diagnóstico urodinâmico 
segundo o tempo de pós-menopausa. Nota-se que o diagnóstico clínico mais freqüente foi de incontinência urinária de esforço nos três grupos, porém, quanto maior o tempo de pós-menopausa, maior a freqüência de urgência miccional. Em relação ao diagnóstico urodinâmico, 93,6\%, 84,6\% e $90,7 \%$, respectivamente, dos grupos A, B e C tiveram incontinência urinária de esforço e $6,4 \%$, $15,4 \%$ e $9,3 \%$ apresentaram instabilidade vesical isolada ou associada a incontinência urinária de esforço.

A análise da fluxometria demonstra que houve diminuição significante do fluxo urinário máximo segundo o tempo de pós-menopausa (Tabela 4). Quanto aos dados obtidos pela cistometria (Tabela 5), observa-se que as pacientes do grupo A apresentaram capacidade vesical no primeiro desejo miccional de $176,29 \mathrm{ml}$, isto é, superior ao valor encontrado no grupo $\mathrm{C}$, que foi de $152,91 \mathrm{ml}$.

São alinhados, na Tabela 6 , os parâmetros referentes ao perfil pressórico uretral, destacandose que não houve variação desse segundo o tempo de pós-menopausa.

Tabela 1 - Dados da anamnese em pacientes menopausadas com queixas urinárias $(n=242)$. Médias e faixas de variação

Dados da

anamnese

$\begin{array}{cc} & \text { Grupos } \\ \text { A } & \text { B }\end{array}$

média (variação) média (variação) média (variação)

\begin{tabular}{lrrrrrr}
\hline idade (anos) & 52,73 & $(44-64)$ & 57,17 & $(45-58)$ & 65,65 & $(48-82)$ \\
$\mathrm{n}^{\mathrm{o}}$ gestações & 6,26 & $(0-16)$ & 5,60 & $(0-16)$ & 4,80 & $(0-18)$ \\
$\mathrm{n}^{\mathrm{o}}$ partos normais & 4,61 & $(0-15)$ & 4,30 & $(0-12)$ & 3,70 & $(0-15)$ \\
$\mathrm{n}^{\mathrm{o}}$ cesárias & 0,5 & $(0-15)$ & 0,31 & $(0-3)$ & 0,16 & $(0-5)$ \\
\hline
\end{tabular}

Grupo A - até 4 anos de menopausa

Grupo B - de 5 a 9 anos de menopausa

Grupo $\mathrm{C}$ - mais de 10 anos de menopausa

Tabela 2 - Diagnósticos clínicos distribuídos pelos grupos. Número e porcentagem em cada grupo

\begin{tabular}{|c|c|c|c|c|c|c|c|c|}
\hline \multirow{3}{*}{$\begin{array}{c}\text { Diagnóstico } \\
\text { Clínico }\end{array}$} & \multicolumn{6}{|c|}{ Grupos } & \multicolumn{2}{|c|}{ Total } \\
\hline & \multicolumn{2}{|r|}{ A } & \multicolumn{2}{|r|}{ B } & \multicolumn{2}{|r|}{$\mathrm{C}$} & \multirow[b]{2}{*}{$\mathrm{n}^{\mathrm{o}}$} & \multirow[b]{2}{*}{$\%$} \\
\hline & $\mathrm{n}^{\mathrm{o}}$ & $(\%)$ & $\mathrm{n}^{\mathrm{o}}$ & $(\%)$ & $\mathrm{n}^{\mathrm{o}}$ & $(\%)$ & & \\
\hline IUE & 38 & $(61,3)$ & 40 & $(76,9)$ & 77 & $(60,2)$ & 155 & $(64,0)$ \\
\hline IURG & 6 & $(9,7)$ & 4 & $(7,7)$ & 12 & $(9,4)$ & 22 & $(9,1)$ \\
\hline IUM & 18 & $(29,0)$ & 8 & $(15,4)$ & 39 & $(30,5)$ & 65 & $(26,9)$ \\
\hline Total & 62 & & 52 & & 128 & & 242 & \\
\hline
\end{tabular}

IUE = incontinência urinária de esforço

IURG = incontinência de urgência

IUM = incontinência urinária mista

Grupo A - até 4 anos de menopausa

Grupo B - de 5 a 9 anos de menopausa

Grupo $\mathrm{C}$ - mais de 10 anos de menopausa
Tabela 3 - Diagnóstico urodinâmico em 242 pacientes menopausadas com queixas urinárias, distribuídas pelos grupos: $A=$ até 5 anos de menopausa; $B=5$ a 10 ano; $\mathrm{C}$ mais que 10 anos.

\begin{tabular}{|c|c|c|c|c|c|c|c|c|}
\hline \multirow{3}{*}{$\begin{array}{l}\text { Diagnóstico } \\
\text { urodinâmico }\end{array}$} & \multicolumn{6}{|c|}{ Grupos } & \multicolumn{2}{|c|}{ Total } \\
\hline & \multicolumn{2}{|r|}{ A } & \multicolumn{2}{|c|}{ B } & \multicolumn{2}{|c|}{$\mathrm{C}$} & \multirow[b]{2}{*}{$\mathrm{n}^{\mathrm{o}}$} & \multirow[b]{2}{*}{$\%$} \\
\hline & $\mathrm{n}^{\mathrm{o}}$ & $(\%)$ & $\mathrm{n}^{\mathrm{o}}$ & $(\%)$ & $\mathrm{n}^{\mathrm{o}}$ & $(\%)$ & & \\
\hline IUE & 58 & $(93,6)$ & 44 & $(84,6)$ & 116 & $(90,7)$ & 218 & $(90,1)$ \\
\hline IURG & 3 & $(4,8)$ & 7 & $(13,5)$ & 8 & $(6,2)$ & 18 & $(7,4)$ \\
\hline IUM & 1 & $(1,6)$ & 1 & $(1,9)$ & 4 & $(3,1)$ & 6 & $(2,5)$ \\
\hline Total & 62 & & 52 & & 128 & & 242 & \\
\hline
\end{tabular}

$\mathrm{IUE}=$ incontinência urinária de esforço

IURG = incontinência do detrusor

IUM = incontinência urinária mista

Grupo $\mathrm{A}$ - até 4 anos de menopausa

Grupo B - de 5 a 9 anos de menopausa

Grupo $\mathrm{C}$ - mais de 10 anos de menopausa

Tabela 4 - Dados da fluxometria em 242 pacientes menopausadas com queixas urinárias distribuídas pelos grupos: $A=$ até 5 anos de menopausa; $B=5$ a 10 ano; $\mathrm{C}$ mais que 10 anos.

Grupos Volume Tempo total de Fluxo urinário Fluxo urinário urinário $(\mathrm{ml})$ micção $(\mathrm{seg})$ médio $(\mathrm{ml} / \mathrm{seg})$ máximo $(\mathrm{ml} / \mathrm{seg})$

\begin{tabular}{lllll}
\hline A & 458.67 & 29.0 & 16.88 & $28.64^{*}$ \\
B & 392.80 & 25.5 & 16.73 & 25.23 \\
C & 393.84 & 24.2 & 14.35 & 24.06
\end{tabular}

* Valores estatisticamente significantes (Kruskal-Wallis)

Tabela 5 - Dados da cistometria em 242 pacientes menopausadas com queixas urinárias, distribuídas pelos grupos: $A=$ até 5 anos de menopausa; $B=5$ a 10 ano; $\mathrm{C}$ mais que 10 anos.

Grupos Resíduo pós- Capacidade vesical do Capacidade vesical miccional $(\mathrm{ml}) \quad 1^{\circ}$ desejo miccional máxima $(\mathrm{ml})$

\begin{tabular}{llll}
\hline A & 458.67 & 29.0 & 16.88 \\
B & 392.80 & 25.5 & 16.73 \\
C & 393.84 & 24.2 & 14.35 \\
\hline
\end{tabular}

* Valores estatisticamente significantes (Kruskal-Wallis)

Tabela 6 - Dados do perfil pressórico uretral em 242 pacientes menopausadas com queixas urinárias, distribuídas pelos grupos. ( $A=$ até 5 anos de menopausa $\mathrm{B}=5$ a 10 ano; $C$ mais que 10 anos).

\begin{tabular}{lcccc}
\hline Grupos & $\begin{array}{c}\text { PMFUch } \\
\left(\mathrm{cmH}_{2} \mathrm{O}\right)\end{array}$ & $\begin{array}{c}\text { CFUch } \\
(\mathrm{cm})\end{array}$ & $\begin{array}{c}\text { PMFUv } \\
\left(\mathrm{cmH}_{2} \mathrm{O}\right)\end{array}$ & $\begin{array}{c}\text { CFUv } \\
(\mathrm{cm})\end{array}$ \\
\hline A & 70,46 & 2,37 & 73,26 & 3,10 \\
B & 57,22 & 2,40 & 60,36 & 3,20 \\
C & 53,08 & 2,20 & 48,88 & 2,80 \\
\hline
\end{tabular}

PMFUch = Pressão máxima de fechamento uretral (bexiga cheia) $\mathrm{CFUch}=$ Comprimento funcional da uretra (bexiga cheia) PMFUv = Pressão máxima de fechamento uretral (bexiga vazia) $\mathrm{CFUv}=$ Comprimento funcional da uretra (bexiga vazia) 


\section{Discussão}

O trato urinário, como se sabe, mantém intima relação com o genital, por terem origem embriológica comum, pela proximidade anatômica e por apresentar, também, receptores esteroídicos e, por isso, responder de modo semelhante à ação hormonal ${ }^{9,18,}$.

Dessa forma, o hipoestrogenismo afeta de maneira evidente o trato urinário, ocasionando alterações tróficas que agravam ou desencadeiam a IUE na pós-menopausa. Nossos resultados não demonstraram diminuição da pressão de fechamento uretral e do comprimento funcional da uretra com o avançar da idade. Esses dados conflitam com os encontrados por alguns autores ${ }^{7,13,17,23,27}$, mas não com que foi observado por Wakavaiachi ${ }^{28}$.

Em relação à capacidade vesical, Diokno et al..$^{13}$ e Gillon et al. ${ }^{16}$ demonstraram ser menor o volume vesical suportado pelas pacientes na pósmenopausa em comparação as no menacme. De igual modo, observamos que as pacientes com mais de 10 anos de pós-menopausa apresentaram, nitidamente, menor capacidade vesical no primeiro desejo miccional do que as com até 5 .

A diminuição da capacidade vesical acarreta sintomas clínicos, tais como urgência miccional, polaciúria e, até mesmo, noctúria. Possivelmente, a deprivação estrogênica atuaria na propiocepção da bexiga, a qual não conseguiria acomodar de modo adequado, maiores volumes.

Portanto, o hipoestrogenismo contribui, provavelmente, para a instalação das alterações urinárias na pós-menopausa. Os nossos resultados demonstraram haver, nesta fase da vida, nitida e significante redução de diversos parâmetros urodinâmicos.

Pode-se concluir, também, que a causa mais comum de perda de urina no climatério é a incontinência urinária de esforço verdadeira, apesar da elevada incidência de instabilidade do detrusor, sobretudo quando em comparação às pacientes no menacme.

\section{SUMMARY}

Purpose: to evaluate the frequency of urinary disorders and variation of the urodynamic parameters according to the time of post-menopause.

Method: two hundred forty-two post-menopausal women with urinary complaints were studied at the Division of Gynecology, Escola Paulista de Medicina, UNIFESP. They were grouped according to the time of post-menopause: group A - up to 4 years; group $B$ - 5 to 9 years and group $C$ - more than 10 years. They were submitted to anamnesis, gynecological examination and urodynamic study. The frequency of urinary alterations and the variation of the urodynamic parameters were analyzed, such as voiding volume; flow time; maximum flow rate, average flow rate; residual urine; vesical capacity at the first desire to void; maximum bladder capacity; maximum urethral closure pressure and functional profile length, with full and empty bladder. The data were statistically analyzed.

Results: the most common clinical diagnosis was stress urinary incontinence in the three groups, but the longer the time of post-menopause, the more frequently urinary urgency was observed. Regarding urodynamic diagnosis, $93.6 \%, 84.6 \%$ and $90.7 \%$ of the patients of the groups $A, B$ and $C$, respectively, presented stress urinary incontinence, while $4.8 \%, 13.5 \%$ and $6.2 \%$ revealed detrusor instability. There was a decrease in the following urodynamic parameters, according to the time of post-menopause: flow time, maximum flow rate and vesical capacity at the first desire to void, and an increase of the residual urine.

Conclusion: in spite of the high incidence of urinary symptoms such as urgency incontinence, stress urinary incontinence was the main urinary problem we have found in post-menopause.

KEY WORDS: Menopause. Urinary disorders. Urodynamics. Incontinence, urinary.

\section{Referências}

1. Aldrighi JM. Distúrbios urogenitais da mulher menopausada: fisiopatologia e aspectos clinicos. J Bras Ginecol 1992. 102 supl.:3-6.

2. Baracat EC, Haidar MA, Rodrigues De Lima G. Síndrome do climatério. Ars Curandi 1989; 22:26-31.

3. Baracat EC, Haidar MA, Rodrigues De Lima G, Simões RD. Síndrome do climatério: aspectos atuais. Ars Curandi 1991; 24:9-16.

4. Bates P, Bradley WE, Glen E, Griffiths D, Melchior H, Rowan D, et al. The standardization of terminology of lower urinary tract function. J Urol 1979; 121:551.

5. Beisland HO, Fossberg E, Moer A, Sander S. Urethral sphincteric insufficiency in postmenopausal females: treatment with phenylpropanolamine and estriol separately and in combination. A urodynamic and clinical evaluation. Urol Int 1984; 39:211-6.

6. Bent AE, Richardson DA, Ostergard DR. Diagnosis of lower urinary tract disorders in postmenopausal patients. Am J Obstet Gynecol 1983; 145:218-22. 
7. Bhatia NN, Ostergard DR. Urodynamics in women with stress urinary incontinence. Obstet Gynecol 1982; 60:552-9.

8. Brincat M, Moniz CF, Studd JWW, Darby AJ, Magos A, Cooper D. Sex hormones and skin collagen content in postmenopausal women. Br Med J 1983; 287:1337-8.

9. Callahan SM, Creed KE. The effects of oestrogens on spontaneous activity and responses to phenylephrine of the mammalian urethra. J Physiol 1985; 358:35-46.

10.Cardozo L. Role of estrogens in the treatment of female urinary incontinence. J Am Geriatr Soc 1990; 38:326-8.

11.Del Castillo EB, Argonz J, Mainini CG. Cytological cycle of the urinary sediment and its parallelism with the vaginal cycle. J Clin Endocrinol 1948; 8:76-87.

12.Del Castillo EB, Argonz J, Mainini CG. Smears from the female urethra and their relationship to smears of the urinary sediment. J Clin Endocrinol Metabol 1949; 9:1362-71.

13. Diokno AC, Wells TJ, Brink CA. Urinary incontinence in elderly women: urodynamic evaluation. J Am Geriatr Soc 1987; 35:940- 6.

14.Faber P, Heidenreich J. Treatment of stress incontince with estrogen in postmenopausal women. Urol Int 1977; 32: 221-3.

15.Fernandes CE, Morita MH, Ferreira JAS, Silva EP, Wehba S. Abordagem dos distúrbios do trato urinário na mulher pós-menopausada. Rev Paul Med 1990; 108:230-5.

16.Gillon G, Savir A, Nissenkorn I, Servadio C. Results and relevance of urodynamic studies in the management of urinary incontinence in women over eighty. Isr Med Sci 1989; 25:25-7.

17.Henriksson L, Andersson KE, Ulmsten U. The urethral pressure profiles in continent and stressincontinent women. Scand J Urol Nephrol 1979; 13:5-10.
18.International Continence Society. The standardisation of terminology of lower urinary tract function. In: Ostergard DR, Bent AE. Urogynecology and urodynamics: theory and practice. 3rd ed. Baltimore: Williams \& Wilkins; 1991. p.545-62

19.Iosif CS, Batra S, Ek A, Astedt B. Estrogen receptors in the human female lower urinary tract. Am J Obstet Gynecol 1981; 141:817- 20.

20.Judge TG. The use of quinestradol in elderly incontinent women, a preliminary report. Gerontol. Clin 11:159-164, 1969.

21.Raz S, Caine M, Zeigler M. The vascular component in the production of intraurethral pressure. J Urol 1972; 108:93-6.

22.Rodrigues De Lima G, Gomes Pinto W, Amoroso JJG, Vasserman J. Considerações sobre a síndrome do climatério. Rev Bras Clin Terap 1981; 10:369-79.

23. Rud T. Urethral pressure profile in continent women from childhood to old age. Acta Obstet Gynecol Scand 1980; 59:331-5.

24.Salmon UJ, Walter RI, Geist SH. The use of estrogens in the treatment of dysuria and incontinence in postmenopausal women. Am J Obstet Gynecol., 42: 845-851, 1941.

25.Schleyer-Saunders E. Hormone implants for urinary disorders in postmenopausal women. J Am Geriatr Soc 1976; 24:337-9.

26.Siegel I, Zelinger BB, Kanter AE. Estrogen therapy for urogenital conditions in the aged. Am J Obstet Gynecol 1962; 84:505-7.

27.Sorensen S. Urodynamic investigations and their reproducibility in healthy postmenopausal females. Scand J Urol Nephrol (Suppl) 1988; 42-7.

28.Wakavaiachi VMB. Análise clínica, urodinâmica e ultra-sonográfica de mulheres continentes e com incontinência urinária de esforço consoante o tempo de menopausa (Tese de Mestrado) São Paulo: Escola Paulista de Medicina; 1997.

\title{
AVISO
}

\section{Comunicamos que a partir de abril, a FEBRASGO enviará aos sócios quites do corrente ano, o selo da FEBRASGO 1999. \\ O Selo deverá ser colado no verso da carteira no local correspondente ao ano de 1999.}

\author{
Caso não tenha recebido sua carteira, entre em contato com a \\ FEBRASGO o mais rápido possível.
}

